\title{
Relationship between Serum Dehydroepiandrosterone (DHEA) Concentration and Physical Fitness after an Exercise Training in Postmenopausal Middle-aged Women
}

\author{
Nayoung Ahn · Kijin Kim* \\ Keimyung University
}

\begin{abstract}
Nayoung Ahn · Kijin Kim. Relationship between Serum Dehydroepiandrosterone (DHEA) Concentration and Physical Fitness after an Exercise Training in Postmenopausal Middle-aged Women. The Journal of Kinesiology, 2017, 19(4): 85-93. [PURPOSE] The purpose of this study was to analyze the relationship between serum dehydroepiandrosterone (DHEA) concentration and physical fitness after exercise in middle-aged women. [METHODS] Subjects were randomly assigned to yoga and elastic band resistance exercise group $(55.21 \pm 3.78 \mathrm{yrs}, \mathrm{n}=11)$ and non-exercise group $(54.10 \pm 2.51 \mathrm{yrs}, \mathrm{n}=7)$. The exercise group participants performed upper and lower extremity exercise three times per week for 12 weeks. Body weight, height, \%fat, serum DHEA concentration, sit-up, grip strength, and one leg stand (with close eyes) were measured on subjects. [RESULTS] Serum DHEA concentration showed an increased trend after exercise training as compared to before exercise training, but no significant difference between before and after exercise training. Also, variables related to physical fitness as sit-up, grip strength, and one leg stand were significantly $(p<.05)$ increased after exercise training as compared to before exercise training. However, serum DHEA concentration and variables related to physical fitness showed no significant relationship. [CONCLUSIONS] Yoga and elastic band resistance exercise training could improve muscular physical fitness, and could be a potential method increasing serum DHEA concentration in middle-aged women, but this possibility is an obscure. In future studies, the intensity of exercise for increasing physical fitness of middle-aged women and the exercise intensity for metabolic function enhancement due to elevated DHEA concentration in blood should be presented differently.
\end{abstract}

Key words : resistance exercise, yoga, middle-aged women, serum DHEA, physical fitness 주요어 : 저항성 운동, 요가, 중년 여성, 혈청 DHEA, 체력

\section{INTRODUCTION}

Postmenopausal women show a rapid decline in ovarian function and estrogen secretion, which is accompanied by weakening of the physiological functions. The steroid sex hormones are secreted by the ovaries, testicles, and adrenal cortex, and these hormones play important roles in physiological response through regular supply to target tissues, such as the bone, liver, cardiovascular system, brain, and skeletal muscle system (Labrie et al., 2005).

In particular, dehydroepiandrosterone (DHEA) is a sex hormone precursor that, together with DHEA sulfatederivative (DHEAS), is the most abundantly produced steroid hormone in humans. Biological functions of DHEAS are responsible for the androgenic effects of adrenarche, such as early pubic and axillary hair growth, adult-type body odor, and increased oiliness of hair and skin. Serum DHEA concentration shows the peak values between 20 and 30 years old, and this value decreases to $50 \%$ level of peak values in the fifth decade, and decreases to $10-20 \%$ level in the ages of 70 - 90 years old (Wend et al., 2012). Due to its rapid decrease in concentration with age and its

* Corresponding author : kjk744@kmu.ac.kr 
important role in declining physiological function during aging (Sato et al., 2014a), DHEA has been considered a major risk factor in relation to the increased prevalence of metabolic disease in old age (Muller et al., 2005).

DHEA is an essential intermediate in the synthesis of sex hormones such as testosterone and estrogen, and so efforts to maintain DHEA levels during aging are an important consideration for health management. Exercise training have been highlighted as a key method to increase levels of DHEA (Sato et al., 2017). Decreased sex hormone levels in aging act as a major risk factor for the elevated prevalence of metabolic disease related to muscle loss (Faulkner et al., 2007). Resistance exercise has been shown to elicit a significant acute hormonal response. It appears that this acute response is more critical to tissue growth and remodelling than chronic changes in resting hormonal concentrations, as many studies have not shown a significant change during resistance training despite increases in muscle strength and hypertrophy. Resistance exercise training is recognized an effective method for tissue regeneration through the increasing of plasma concentration of steroid hormones (Kraemer et al., 2017) and DHEA (Sato et al., 2008), which leads to the increase of muscle mass, the level of antioxidant, and anti-inflammatory factors in muscles (Gasier et al., 2011). In this regard, Sato et al. (2014a) implemented resistance training in elderly male subjects for the increased secretion of steroid hormone. Meanwhile, P öllänen et al. (2015) found a strong correlation between intramuscular steroid hormone levels and muscular strength in women, and reported that the concentration of steroid hormones decreased dramatically after menopause. Legrain \& Giraed (2003) reported that plasma DHEA levels were associated with impaired physical function in the elderly, and the prevalence and mortality of age-related degenerative disease, emphasizing the importance of using exercise to maintain the concentration of sex hormones for health management in the elderly. Aging is associated with a decrease in circulating DHEA, which results in a decrease in physical metabolic function and an increase in metabolic disease (Montero-Fern ández \& Serra- Rexach, 2013). Exercise training can be an effective method for the prevention of metabolic disease. However, the majority of research showing the effects of exercise on DHEA protein expression in skeletal muscle (Aizawa et al., 2007) has reported a positive effect in animal experiments, and various studies have been conducted on the physiological importance and mechanisms of DHEA in aging (Di Blasio et al., 2017). Therefore we could not suggest the critical or enough detail effects of exercise training program on the prevention of aging process based on exercise-mediated mechanisms of change in DHEA.

In postmenopausal middle-aged women in particular, muscle loss and weakness lead to a reduction in immune function, and present a considerable risk of osteoporosis and metabolic disease (Ahn \& Kim, 2015). Because menopause is the actual point of aging process, examining the change in serum DHEA concentration after performing a resistance exercise program in menopausal women could provide important information to analyze the effects of exercise. Yoga gives elasticity to muscles, tones tendons and ligaments, reduces fat and slows weight gain. Improved posture is also an important aspect of yoga. Yoga could reverse the aging process by the positive impact the practice has upon the body. It Rao et al. (2015) suggested mind and body therapeutic program with yoga improved the human well-being related to cellular and molecular function. Therefore, we aimed to analyze the association between serum DHEA concentration and changes in physical fitness parameters after perform an exercise training in postmenopausal middle-aged women. 


\section{METHODS}

\section{Subjects}

The subjects in this study consist of postmenopausal women who have no prior experience of specialist exercise, are first starting yoga and resistance exercise training, and have shown no specific findings in medical interviews or examinations in the last 6 months. The exercise training program was conducted 3 times per week for 12 weeks, steadily increasing the intensity. Subjects participated voluntarily, after receiving a thorough explanation of the research aims and method, and completing a consent form. The subjects' physical characteristics are presented in $<$ Table 1> The study protocol was in compliance with the Declaration of Helsinki.

Table 1. Characteristic of subjscts

\begin{tabular}{ccccc}
\hline Group(n) & Age (yrs) & $\begin{array}{c}\text { Height } \\
(\mathrm{cm})\end{array}$ & $\begin{array}{c}\text { Weight } \\
(\mathrm{kg})\end{array}$ & \%Fat \\
\hline \hline \multirow{2}{*}{ Ex(11) } & 55.21 & 156.72 & 57.72 & 31.44 \\
& \pm 3.78 & \pm 3.45 & \pm 3.83 & \pm 4.91 \\
Control(7) & 54.10 & 156.10 & 56.61 & 28.88 \\
& \pm 2.51 & \pm 4.78 & \pm 4.36 & \pm 5.40 \\
\hline
\end{tabular}

Ex; Yoga and elastic band resistance exercise; \%Fat: \% body fat mass

\section{Yoga and resistance band exercise program}

Accounting for the subjects' physical characteristics and their beginner status, yoga and resistance band exercise were selected for exercise training program. The exercise training program was implemented 3 times per week for 12 weeks; yoga and resistance band exercise intensity was gradually increased every 4 weeks (changing the band color and/or increasing the number of repetitions). Using Borg's (Borg, 1982) rating scale of perceived exertion (RPE), RPE was measured every 4 weeks, and the exercise intensity was steadily increased to maintain an RPE of 12 - 16 with the check of verbal testing during exercise $<$ Table $2>$.

\section{Measured variables and methods}

\section{Body composition \& physical fitness parameters}

The subjects' height (TKK, Japan), body weight (TKK, Japan), and \%fat (Skinfold caliper, Skyndex U.S.A) (Siri, 1961) were measured. Muscular strength was measured using a hand dynamometer (TKK, Japan) to measure grip strength. Muscular endurance was measured using sit-ups. Balance was measured using the one-leg standing balance test with eyes closed.

\section{Analysis of serum DHEA concentration}

Subjects were instructed to fast for at least 12 hours, and then to rest for 30 minutes before $3 \mathrm{ml}$ of venous

Table 2. Yoga and elastic band resistance exercise program

\begin{tabular}{clcc}
\hline Variables & \multicolumn{1}{c}{ Contents } & Intensity & $\begin{array}{c}\text { Time } \\
\text { (mins) }\end{array}$ \\
\hline \hline Warm up & Stretching \& abdominal respiration & - & 10 \\
\hline Yoga & $\begin{array}{l}\text { Cat position, Twist position, Lifting legs, Sit \& reach, Core holding } \\
\text { position, Variation position }\end{array}$ & RPE 12 14 & 30 \\
\hline $\begin{array}{c}\text { Elastic band } \\
\text { resistance } \\
\text { exercise }\end{array}$ & $\begin{array}{l}\text { Seated rows, overhead or military press, Hip flexion, Calf raises, } \\
\text { Hip extension }\end{array}$ & $\begin{array}{c}10 \times 3 \text { sets, } \\
\text { Yellow, red \& green } \\
\text { color band } \\
\text { RPE } 14 \sim 16\end{array}$ & 20 \\
\hline Cool down & Stretching \& breathing & RPE $8 \sim 10$ & 10 \\
\hline
\end{tabular}


blood was collected from the antecubital vein. Blood was always collected between 9.00 and 10.00am to control for circadian rhythm. The serum was isolated by spinning for 5 minutes in a centrifuge at 3000rpm, before storing at $-20^{\circ} \mathrm{C}$. The serum DHEA concentration was analyzed using a COBRA 5010 II, Quantum analyzer with the Coat-A-Count DHEA-sulfate (DPC; Diagnostic Products CO.) reagent.

\section{Statistical analysis}

The SPSS 18.0 statistics program was used to calculate the mean and standard deviation for each measured variable. A repeated 2-way ANOVA was used to test for significant differences between groups and time points. In the case of a significant interaction between group and time, an independent t-test for between-group comparisons and a paired t-test for before-after comparisons in each group were conducted for a post-hoc. A Pearson's correlation analysis was performed to examine the relationship between serum DHEA concentration and physical fitness. A significance level of $p<.05$ was used.

\section{RESULTS}

\section{Changes in serum DHEA and physical fitness with 12 weeks of exercise}

After 12 weeks of yoga and resistance band exercise, the serum DHEA concentration showed an increasing trend $(p=0.191)$ in the exercise group, but there was no significant difference. Grip strength, sit-ups, and one-leg standing balance test (eyes closed) showed a significant interaction between group and time $(p<.05)$; post-hoc testing revealed that all physical fitness-related variables increased significantly in the exercise group after exercise $(p<.01)$, but there was no significant change in the control group <Table $3>$.

\section{Correlation between serum DHEA concentration and physical fitness}

Although there was a positive correlation between serum DHEA concentration and \%fat, it was not statistically significant $<$ Figure $1>$.

Table 3. Changes of DHEA concentration and physical fitness after 12 week exercise in postmenopausal middle-aged women (M $\pm S D$ )

\begin{tabular}{|c|c|c|c|c|c|c|}
\hline \multirow[b]{2}{*}{ Variables } & \multirow[b]{2}{*}{ Group } & \multirow[b]{2}{*}{ Pre } & \multirow[b]{2}{*}{ Post } & \multicolumn{3}{|c|}{ F-value } \\
\hline & & & & Group & Time & $\begin{array}{l}\text { Group } \\
\times \text { Time }\end{array}$ \\
\hline \multirow{2}{*}{$\begin{array}{l}\text { Serum DHEA } \\
(\mathrm{mg} / \mathrm{dL})\end{array}$} & Ex $(n=11)$ & $118.71 \pm 22.41$ & $128.05 \pm 16.02$ & \multirow{2}{*}{0.135} & \multirow{2}{*}{0.489} & \multirow{2}{*}{0.013} \\
\hline & Control $(\mathrm{n}=7)$ & $102.98 \pm 19.93$ & $99.06 \pm 13.66$ & & & \\
\hline \multirow{2}{*}{$\begin{array}{l}\text { Grip strength } \\
(\mathrm{kg})\end{array}$} & Ex $(n=11)$ & $19.12 \pm 2.57$ & $23.96 \pm 3.95^{*}$ & \multirow{2}{*}{4.578} & \multirow{2}{*}{$13.657^{*}$} & \multirow{2}{*}{$6.009^{*}$} \\
\hline & Control $(\mathrm{n}=7)$ & $18.65 \pm 1.26$ & $19.98 \pm 3.35$ & & & \\
\hline \multirow{2}{*}{$\begin{array}{l}\text { Sit-up } \\
\text { (times) }\end{array}$} & Ex $(n=8)$ & $15.25 \pm 5.42$ & $20.58 \pm 6.85^{*}$ & \multirow{2}{*}{1.351} & \multirow{2}{*}{$4.265^{*}$} & \multirow{2}{*}{$9.714^{*}$} \\
\hline & Control $(\mathrm{n}=4)$ & $16.65 \pm 9.93$ & $10.36 \pm 5.61$ & & & \\
\hline \multirow{2}{*}{ One leg stand (sec) } & Ex $(n=11)$ & $3.37 \pm 4.89$ & $17.65 \pm 8.79 * *$ & \multirow{2}{*}{0.340} & \multirow{2}{*}{$5.930^{*}$} & \multirow{2}{*}{$6.213^{*}$} \\
\hline & Control $(\mathrm{n}=7)$ & $6.00 \pm 5.70$ & $9.27 \pm 6.26$ & & & \\
\hline
\end{tabular}

*: $p<0.05, * *: p<0.01$; DHEA: dehydroepiandrosterone 


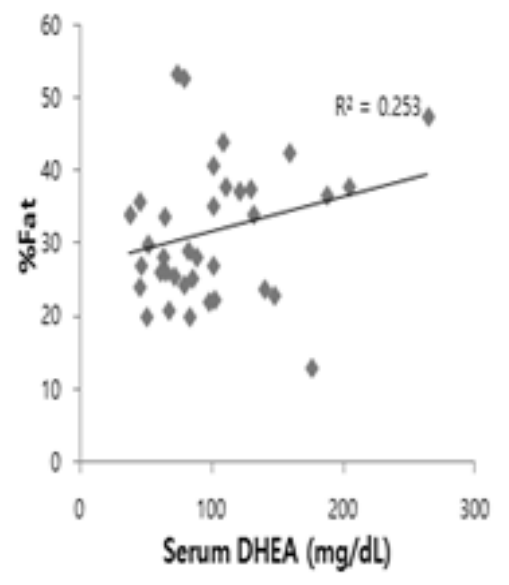

Figure. 1. Correlation between serum DHEA conœentration and $\%$ fat after 12 wk exercise in post-menopausal middle- aged women $(n=36)$

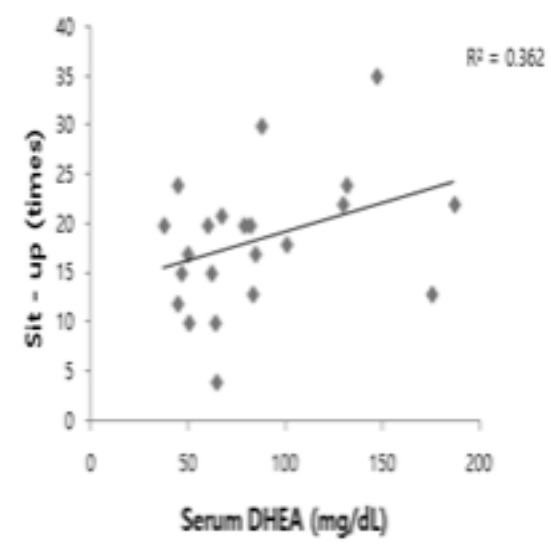

Figure. 2. Correlation between serum DHEA concentration and sit-up after 12 wk exercise in post-menopausal middle-aged women $(n=24)$

Meanwhile, among physical fitness parameters, sit-ups showed a positive correlation with DHEA, but this was not statistically significant $<$ Figure $2>$, and there were also no significant relationships of serum DHEA with grip strength <Figure $3>$ or one-leg standing balance $<$ Figure $4>$.

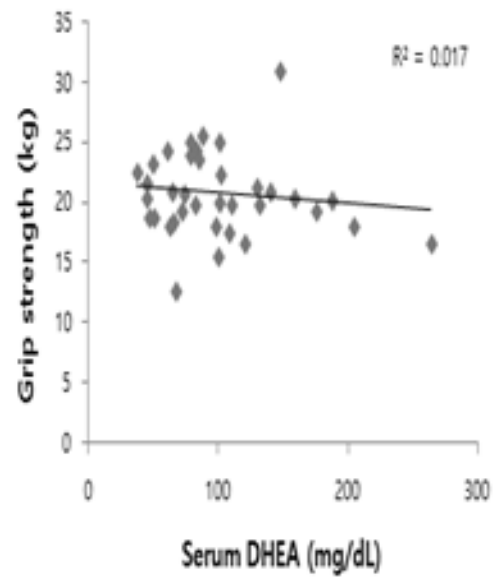

Figure. 3. Correlation between serum DHEA concentration and grip strength after 12 wk exercise in postmenopausal middle-aged women $(n=36)$

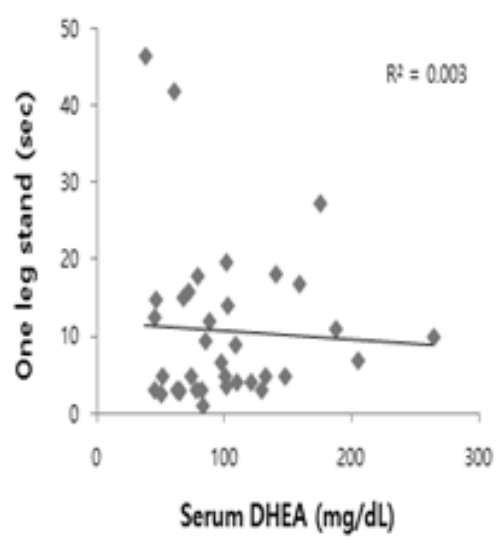

Figure. 4. Correlation between serum DHEA concentration and one-leg stand after 12 wk exercise in postmenopausal middle-aged women $(n=36)$

\section{DISCUSSION}

In middle-aged women, after the menopause begins from the mid-40s, sex hormone levels gradually decrease and do not meet the required levels; this is accompanied by the use of DHEA from the adrenal glands. As a result, metabolic function declines considerably in the menopause, leading to an increase in the prevalence of metabolic disease, and a large decrease in serum DHEA 
levels (Pöllänen et al., 2011). DHEA is converted to testosterone by 3ß-hydroxysteroid dehydrogenase (HSD) during synthesis, and 17ß-HSD and testosterone are converted to $5 \mathrm{a}$-dihydrotestosterone (DHT) by $5 \mathrm{a}$ -reductase (Labrie et al., 2005). In particular, DHEA is known to inhibit cortisol, and to play important roles in reducing body fat, enhancing immune function, and protecting against cardiovascular disease and osteoporosis (Muller et al., 2005).

The reason DHEA can replace sex hormones is because it can make estrogen in the musculoskeletal system by binding with the enzyme aromatase (aromatase cytochrome P450 enzyme) (Longcope et al., 1978). Thus, controlling DHEA levels is a key part of health management to prevent the decline of metabolic function in postmenopausal women in their 40s and older. According to recent analysis in rats, DHEA from cultured myocytes was able to synthesize estrogen, estradiol, and DHT. And previous studies reported that muscle mass positively associated with the secretion of sex hormones (Aizawa et al., 2007; Sato et al., 2008). However, efforts are still required to obtain clearer results on the mechanisms of steroid hormone loss and muscle-related changes in sex hormones during the aging process.

Sato et al. (2014a) performed 12 weeks of isokinetic knee exercises in 13 elderly individuals and 6 young adults, and analyzed the vastus lateralis muscle, they reported a significant decrease in DHEA secretion from muscles and DHT expression in the elderly group compared to the young group. However, a follow-up study using 12 weeks of resistance training reported a significant elevation of DHEA and DHT expression, and a very significant increase in steroidogenesis-related enzymes such as HSD, 17-HSD, and 5-reductase, highlighting the importance of resistance training to maintain levels of sex hormone secretion during aging ( $\mathrm{P}$ öllänen et al.,
2011; Sato 2014b). Pöllänen et al. (2011) reported a significant correlation between muscular strength and serum DHEA per unit cross-sectional area (CSA) of muscle. In addition, in a follow-up study, Pöllänen et al. (2015) reported that intramuscular steroid sex hormone secretion (testosterone, 17ß-estradiol, DHT \& DHEA) was correlated with muscular strength and explosive muscle power, and that it was not correlated with the muscle CSA. However, Vingren et al. (2008) performed short-term resistance training in younger individuals and found no significant change in muscular sex steroid hormone secretion, meaning that there are conflicting results for the effects of resistance training.

In this regard, the present study also found significant increases in the physical fitness-related variables of grip strength, sit-ups, and one-leg standing balance (eyes closed) after performed 12 weeks of exercise, but there was no statistically significant change in serum DHEA concentration. Among factors that might have caused this result, the suitability of the exercise intensity needs to be considered in middle-aged women with no experience of specialist exercise. An intensity of RPE 12 - 16 for yoga and resistance band exercise is considered a moderate intensity for the improvement of physical fitness, but it is possible that it corresponds to low-to-moderate intensity in terms of its effect on steroid hormone secretion. As RPE is difficult to control for an application of the exact exercise intensity during exercise training, we should consider the check of heart rate for exact control of exercise intensity at next research. Compared to a previous study that produced a large change by using isokinetic exercise, some difference in the exercise intensity is expected. In addition, there could be temporal and physical differences between the stage of DHEA protein expression in tissues and the level of DHEA circulating in the blood (Morgan et al., 2013). Postmenopausal women strongly need 
resistance and weight training to increase muscle mass and improve muscular strength, but the suitability of exercise intensity needs to be carefully considered for more effect on exercise performance and physical fitness. Furthermore, in order to accurately analyze the effects, it is important to account for the possibility of a temporal and physical difference between protein expression at the cell level and in the concentration of hormones in the circulation.

In studies with conflicting results for DHEA changes after exercise, the role of cortisol needs to be considered. DHEA alleviates the adverse effects of cortisol (Allolio \& Arlt, 2002), and excessively high DHEA levels are known to cause ovarian cysts in females, and to mimic the effects of female hormones in males (Granger et al., 1999). In adrenal insufficiency, DHEA restores DHEA/DHEAS and androstenedione levels, reduces total cholesterol, improves well-being, sexual satisfaction and insulin sensitivity, and prevents loss of bone mineral density (Rutkowski et al., 2014). The increase in DHEA concentration during exercise is thought to be due to the neurohormone antiglucocorticoid, which has the opposite action to cortisol. In stressful situations, the DHEA concentration increases together with the cortisol concentration. And the DHEA concentration alleviates the adverse effects of cortisol, producing anti-epileptic, anti-depressive, and anxiolytic effects (Morrow et al., 1995). Di Blasio et al. (2017) reported that postmenopausal women show a relative increase in cortisol and a decrease in DHEAS, but that this can be expected to improve with walking or other exercise training. Although the result was not statistically significant in our study, we think that the trend for increase in DHEA after exercise is an evidence to emphasize the importance of exercise programs for the maintenance of DHEA levels in postmenopausal women. Our study did not show a significant correlation between serum DHEA concentration and parameters of physical fitness. This may be seen as the result of differences in hormone concentration in the blood and expression in actual tissues, which is due to the action of hormone intermediates that have a large effect on metabolic activity, including cortisol and DHEA expression at the cell level. Another possible cause is the differences in the time of hormone expression, but further research $\mathrm{w}$ ill be required to obtain a more definite result.

Pöllänenet et al. (2011) found that intramuscular DHT expression was higher in elderly women than in younger women, and so claimed that there was no correlation with age. However, Sato et al. (2014a; 2014b) reported that intramuscular DHEA, free-testosterone, and DHT expression were higher in younger males than in elderly males, indicating that gender differences have a major impact on the level of steroid hormone secretion. Although not certain, it is possible that differences in the sex hormone systems of men and women mean that the steroid sex hormone response to exercise is more effective in males than in females. On the other hand, an effective correlation analysis was not possible in the present study due to the small number of subjects. In future research, it will be important to increase the number of subjects and to compare the effects of exercise treatment under the same conditions between males and females.

\section{CONCLUSIONS}

This study performed a 12-week resistance training program using yoga and resistance band in postmenopausal middle-aged women, and analyzed the serum DHEA concentration, parameters of physical fitness, and their correlations after exercise training. After exercise training, physical fitness 
improved significantly, and serum DHEA showed an increasing trend that was not statistically significant. This highlights the importance of detail consideration of exercise training to maintain appropriate DHEA levels in postmenopausal women. There was no significant correlation between serum DHEA concentration and parameters of physical fitness, which is thought to show the importance of using the appropriate exercise intensity.

\section{REFERENCES}

Ahn, N. Y., \& Kim K. J. (2015). Comparison of training effects on physical fitness metabolic syndrome-related risk factors according to changes of body fat or cardiopulmonary function after exercise training between pre and post-menopausal middle-aged women. Exercise Science, 24(3): 253-265.

Aizawa, K., Iemitsu, M., Maeda, S., Jesmin, S., Otsuki, T., Mowa, C. N., Miyauchi, T., \& Mesaki, N. (2007). Expression of steroidogenic enzymes and synthesis of sex steroid hormones from DHEA in skeletal muscle of rats. American Journal of Physiology Endocrinology, and Metabolism, 292: E577-E584.

Allolio, B., \& Arlt, W. (2002). DHEA treatment: myth or reality? Trends in Endocrinology and Metabolism, 13(7): 288-294.

Borg, G. A. V. (1982). Psychophysical bases of perceived exertion. Medicine and Science in Sports and Exercise, 14: 377-381.

Di Blasio, A., Izzicupo, P., Di Baldassarre, A., Gallina, S., Bucci, I., Giuliani, C., Di Santo, S., Di Iorio, A., Ripari, P., \& Napolitano, G. (2017). Walking training and cortisol to DHEA-S ratio in postmenopausal: An intervention study. Women \& Health, 57(1): 1-16.

Faulkner, J. A., Larkin, L. M., Claflin, D. R., \&
Brooks, S. V. (2007). Age-related changes in the structure and function of skeletal muscles. Cinical Experimental Pharmacology and Physiology, 34: 1091-1096.

Gasier, H. G., Riechman, S. E., Wiggs, M. P., Buentello, A., Previs, S. F., \& Fluckey, J. D. (2011). Cumulative responses of muscle protein synthesis are augmented with chronic resistance exercise training. Acta Physiology (Oxford), 201: 381-389.

Granger, D. A., Schwartz, E. B., Booth, A., \& Curran, M. (1999). Assessing dehydroepiandrosterone in saliva: a simple radioimmunoassay for use in studies of children, adolescents and adults. Psychoneuroendocrinology, 24: 567-579.

Kraemer, W. J., Ratamess, N. A., \& Nindl, B. C. (2017). Recovery responses of testosterone, growth hormone, and IGF-1 after resistance exercise. Journal of Applied Physiology, 122: 549-558.

Labrie, F., Luu-The, V., Bélanger, A., Lin, S. X., Simard, J., Pelletier, G., \& Labrie, C. (2005). Is dehydroepiandrosterone a hormone? Journal of Endocrinology, 187: 169-196.

Legrain, S., \& Girard, L. (2003). Pharmacology and therapeutic effects of dyhydroepiandrosterone in older subjects. Drugs Aging, 20(13): 949-967.

Longcope, C., Pratt, J. H., Schneider, S. H., \& Fineberg, S. E. (1978). Aromatization of androgens by muscle and adipose tissue in vivo. Journal of Clinical Endocrinology and Metabolism, 46: 146-152.

Montero-Fernández, N., \& Serra-Rexach, J. A. (2013). Role of exercise on sarcopenia in the elderly. European Journal of Physical Rehabilitation and Medicine, 49: 131-143.

Morgan, F. M., Chen, J., Gee, N. A., Lohstroh, P., \& Lasley, B. L. (2013). Dehydroepiandrosterone sulfate (DHEAS) levels reflect endogenous LH production and response to human chorionic gonadotropin (hCG) challenge in the older female macaque (Macaca fascicularis). Menopause, 20(3): 329-335. 
Morrow, A. L., Devaud, L. L., Purdy, R. H., \& Paul, S. M. (1995). Neuroactive steroid modulators of the stress response. New York Academic Science, 771: 257-272.

Muller, M, Grobbee, D. E., den Tonkelaar, I., Lamberts, S. W., \& van der Schouw, Y. T. (2005). Endogenous sex hormones and metabolic syndrome in aging men. Journal of Clinical Endocrinology and Metabolism, 90: 2618-2623.

Pöllänen, E., Kangas, R., Horttanainen, M., Niskala, P., Kaprio, J., Butler-Browne, G., Mouly, V., Sipilä, S., \& Kovanen, V. (2015). Intramuscular sex hormones are associated with skeletal muscle strength and power in women with different hormonal status. Aging Cell, 14: 236-248.

Pöllänen, E., Sipilä, S., Alen, M., Ronkainen, P. H. A., Ankarberg-Lindgren, C., Puolakka, J., Suominen, H., Hämäläinen, E., Turpeinen, U., Konttinen, Y. T., \& Kovanen, V. (2011). Differential influence of peripheral and systemic sex steroids on skeletal muscle quality in pre- and postmenopausal women. Aging Cell, 10: 650-660.

Rao, K. S., Chakrabarti, S. K, Dongare, V. S., Chetana, K, Ramirez, C. M, Koka, P. S., \& Deb, K. D. (2015). Antiaging effects of an intensive mind and body therapeutic program through enhancement of telomerase activity and adult stem cell counts. Journal of Stem Cells, 10(2): 107-125.

Rutkowski, K., Sowa, P., Rutkowski,-Talipska, J., Kuryliszyn-Moskal, A., \& Rutkowski, R. (2014). Dehydroepiandrosterone (DHEA): hypes and hopes. Drug, 74(11): 1195-1207.

Sato, K, Fujita, S., \& Iemitsu, M. (2017). Dioscorea esculenta-induced increase in muscle sex steroid hormones is associated with enhanced insulin sensitivity in a type 2 diabetes rat model. The Official Journal of the Federation of American Societies for Experimental Biology, 31(2); 793-801.

Sato, K., Iemitsu, M, Aizawa, K., \& Ajisaka, R. (2008). Testosterone \& DHEA activate the glucose metabolism-related signaling pathway in skeletal muscle. American Journal of Physiology, 294: E961-E968.

Sato, K., Iemitsu, M., Matsutani, K., Kurihara, T., Hamaoka, T., \& Fujita, S. (2014a). Resistance training restores muscle sex steroid hormone steroidogenesis in older men. The Official Journal of the Federation of American Societies for Experimental Biology, 28: 1891-1897.

Sato, K., Samocha-Bone, D., Handelsman, D. J., Fujita, S., Wittert, G. A., \& Heilbronn, L. K. (2014b). Serum sex steroids and steroidogenesisrelated enzyme expression in skeletal muscle during experimental weight gain in men. Diabetes Metabolism, 40: 439-444.

Siri, S. E. (1961). Body composition from fluid spaces and density: analysis of methods. In: Brozek J, Henschel A. Techniques for measuring body composition. Washington DC: National Academy of Sciences, National Research Counncil, 223-244.

Vingren, J. L., Kraemer, W. J., Hatfield, D. L., Anderson, J. M., Volek, J. S., Ratamess, N. A., Thomas, G. A., Ho, J. Y., Fragala, M. S., \& Maresh, C. M. (2008). Effect of resistance exercise on muscle steroidogenesis. Journal of Applied Physiology, 105: 1754-1760.

Wend, K., Wend, P., \& Krum, S. A. (2012). Tissue-specific effects of loss of estrogen during menopause and aging. Frontier Endocrinology (Lausanne), 3: 19. 
